\title{
Iluminar as Vidas Longas em Meio Rural: o Recurso à Música na Promoção do Envelhecimento Ativo e Saudável
}

Shining Long Lives in Rural Areas: Music to Promote Active and Healthy Ageing

\section{Lília Maria Moreira Pinto}

Santa Casa da Misericórdia do Marco de Canaveses, Faculdade de Medicina da Universidade do Porto psicologia@scmmarco.com

\section{Francisca Estrela Resende}

Santa Casa da Misericórdia do Marco de Canaveses

terapiaocupacional@scmmarco.com

\section{André Valente Ramos}

Santa Casa da Misericórdia do Marco de Canaveses

andreramos.saas@scmmarco.com

Maria Amélia Ferreira

Santa Casa da Misericórdia do Marco de Canaveses, Faculdade de Medicina da Universidade do Porto

mameliaferreira@med.up.pt

Conflito de interesses: nada a declarar. Financiamento: nada a declarar. 


\section{RESUMO}

É através da articulação indissociável da saúde e da área social que a Santa Casa da Misericórdia de Marco de Canaveses (SCMMC) tem inovado em cuidados de saúde especializados na população idosa, fragilizada, empobrecida e isolada em tempo COVID19. Este princípio motivou a elaboração de dois Projetos de Saúde Social, baseados na arte musical - "Bairros Saudáveis: Incluir para Melhorar" e "Musicalidade" - cujos objetivos focam o processo de Envelhecimento Ativo e Saudável. Através de intervenções diversas, com recurso à música, procurar-se-á fortalecer o sentido de pertença na comunidade dos beneficiários idosos a residir no próprio domicílio e dos idosos inscritos em respostas sociais do concelho de Marco de Canaveses, através de uma intervenção de proximidade, focada na prevenção e promoção da saúde mental. Construindo-se uma intervenção personalizada em meio rural, no decurso destes projetos, projeta-se que os benefícios das atividades musicais na população idosa permitam a diminuição do isolamento e do sentimento de solidão; a melhoria na perceção sobre a qualidade de vida e bem-estar psicológico; e diminuição da sintomatologia depressiva, ansiosa e dos níveis de stress. É com um novo olhar sobre o envelhecimento em casa (in place) e na comunidade (in community) aliando os efeitos positivos da música, enquanto terapia, que se tenciona progredir na promoção do envelhecimento sustentável, enquanto referência de boas práticas sociais e de saúde.

Palavras-Chave: Envelhecimento ativo e saudável, Intervenção pela música, Isolamento Social, Solidão

\section{ABSTRACT}

It is through the inseparable articulation of health and the social fields that the Santa Casa da Misericórdia de Marco de Canaveses (SCMMC) has been innovating in the specialized health care on the elderly, fragile, impoverished and isolated population through COVID19 times. This principle motivated the construction of two Social Health Projects, based on musical art - "Bairros Saudáveis: Incluir Para Melhorar" and "Musicalidade" - whose aim is focused on the process of Active and Healthy Aging. Through different types of interventions, using music, we will seek to strengthen the belongingness in the community of old people living in their own homes and those included in social responses in the municipality of Marco de Canaveses, through an intervention based on proximity and focused on the prevention and promotion of mental health. Building a personalized intervention in rural areas, in the course of these projects, it is expected that the benefits 
of musical activities in the elderly population allow the reduction of isolation and the feeling of loneliness; improving the perception of quality of life and psychologic well-being; and decreased depressive, anxious symptomatology and stress levels.It is with a new perspective for the process of aging in place and aging in community, combining the positive effects of music as a therapeutic approach, that it is intended to progress in the promotion of sustainable aging as a reference of good social and health practices.

Keywords: Active and Healthy Ageing, Intervention using music, Social Isolation, Loneliness 


\section{ENQUADRAMENTO TEÓRICO}

0 aumento do número de pessoas idosas demonstrado em todo o mundo, gera importantes desafios sociodemográficos que implicam a tomada de decisões por parte da Organização Mundial da Saúde e dos Governos. Está hoje demonstrado que as medidas promotoras do envelhecimento ativo e saudável são referência nuclear de boas práticas de intervenção neste grupo etário.

Partindo da importância da promoção do envelhecimento sustentável e no âmbito da Responsabilidade Social da Santa Casa da Misericórdia do Marco de Canaveses (SCMMC), esta entidade assume como fundamental a intervenção na população mais velha através de uma abordagem que congregue medidas na saúde e na área social. Com a execução de diversos programas de intervenção desenhados para cumprir com esta relação saúdesocial, em especial, pelo caráter inovador, "Bairros Saudáveis: Incluir para melhorar" e "Musicalidade", promovem-se através de intervenção musical, a estimulação das competências cognitivas, motoras e sensoriais, maximizando a participação e a capacidade funcional de cada idoso, centrando o seu papel numa lógica de diminuição do isolamento social e do sentimento de solidão.

\subsection{Envelhecimento e a pandemia COVID-19}

Em Portugal, como nos restantes países a nível mundial, tem-se assistido ao aumento significativo da esperança média de vida, o que pressupõe que haja gente a viver cada vez mais anos. 0 índice de envelhecimento (número de pessoas com 65 e mais anos por 100 jovens menores de 15 anos) do nosso país coloca-o entre os primeiros países "mais velhos" da União Europeia. Este notório crescimento da população mais velha coloca exigências cada vez mais urgentes e complexas sobre os Sistemas de Saúde e os Serviços Sociais, aumentando os desafios para os sistemas de previdência em todo o mundo (UN, 2015; WHO, 2015).

A distribuição do indicador de envelhecimento pela tipologia de áreas urbanas mostra, ainda, que a proporção de população residente com 65 ou mais anos é mais elevada nas áreas predominantemente rurais do que nas áreas predominantemente urbanas, sendo 0 caso concreto de Marco de Canaveses (INE, 2021).

Com uma população cada vez mais envelhecida é facilmente demonstrável que as alterações inerentes ao envelhecimento desencadeiam problemas de saúde, que restringem a realização de atividades diárias, pela perda da independência física, social e com tendência a constantes mudanças emocionais. As alterações subjacentes ao envelhecimento podem levar não só a um declínio estrutural e funcional dos sistemas 
nervoso e musculoesquelético (Goldspink \& Harridge, 2004) - associadas a aumento do risco de quedas, fraturas, imobilidade, declínio funcional e, por sua vez, dependência mas também a alterações no sistema sensorial (Gai et al., 2009; Lewis, 2003) e da reserva cognitiva - como diminuição das funções executivas, notória na dificuldade de tomada de decisão ou no planeamento de atividades (Bherer, 2015). Embora algumas alterações da saúde das pessoas mais velhas sejam genéticas, os contextos físicos e sociais revestemse de grande importância, assim como as suas caraterísticas pessoais (ex: etnia, género, nível socioeconómico) no processo de ficar mais velho (WHO, 2021).

Aos declínios na saúde a que os mais velhos estão sujeitos, no decurso do envelhecimento, somam-se ainda problemas de autonomia e independência para a realização das atividades diárias, passando a depender de outras pessoas (Kazazi et al., 2018). Esta redução do empowerment e das competências funcionais dos idosos não deve ser fator limitador da participação social, pois sendo-o tenderá a aumentar as situações de solidão e isolamento social. A autonomia pessoal e a capacidade de fazer escolhas são importantes para os idosos, na mesma medida que é a sua perceção de saúde (Shearer et al., 2012).

Neste contexto, decorrente da instalação da pandemia da COVID-19 e as respetivas restrições ao normal funcionamento da rotina diária e da liberdade de cada pessoa vieram exacerbar, numa esfera pessoal, os sentimentos de isolamento e incapacidade. Diversos estudos demonstraram que a esfera socioemocional da pandemia (restrição nas saídas de casa, nos contactos com amigos e familiares, encerramento de Centros de Convívio/ Dia, entre outros) foi uma das mais fortemente afetadas e que é motivo para significativas repercussões danosas na saúde física e mental das pessoas (Whitehead \& Torossian, 2020; Wu, 2020). 0 grupo etário de 65 e mais anos foi um dos grupos de risco desde logo definidos pela Direção-Geral de Saúde, pela particular suscetibilidade de contrair a doença. Visto que este grupo representa uma grande percentagem de toda a população portuguesa, é certamente o que mais "sofreu" com a presença da pandemia e suas consequências. Já é por si só o conjunto da população mais só, que normalmente reside em locais mais rurais e, com a pandemia, ainda teve que ficar mais protegido e isolado na proteção da COVID-19.

\subsection{Solidão e Isolamento Social}

0 envelhecimento é um processo evolutivo onde há que ter em consideração as relações entre os múltiplos aspetos que o integram, como a vida familiar, o emprego, a educação, a vida sociocultural, a saúde e a qualidade de vida (Ferreira, 2011). No entanto podem surgir barreiras a este envelhecimento que podem ser fruto apenas de como a sociedade encara os mais velhos, o que influencia em maior escala as decisões e prioridades atribuídas a este grupo da sociedade. A forma como se encara o envelhecimento deve ser 
um ponto de partida para a promoção do envelhecimento ativo e saudável, com participação na vida social e comunitária (Rede Nacional de Cidades Amigas das Pessoas Idosas), contrariando os níveis elevados dos sentimentos de solidão e isolamento sentidos pelos que vão envelhecendo.

0 isolamento social e o sentimento de solidão tiveram um impacto e notoriedade maiores durante a pandemia COVID-19, com as restrições de mobilidade e as medidas de distanciamento social adotadas para conter a propagação do vírus, o que fez valorizar estas emoções como predisposição para outras doenças que afetam a saúde mental e física. Acresce ainda que a exclusão social da população idosa se reflete numa sobrecarga socioeconómica que tem impacto negativo direto nos custos associados aos Sistemas Nacional de Saúde e Segurança Social (Longman et al., 2013). Paralelamente, contribui para um empobrecimento da saúde física e mental pelos efeitos diretos que os sentimentos de solidão acarretam nos idosos (Losada et al., 2012) e que se repercute no progressivo declínio do seu funcionamento cognitivo (Marioni et al., 2012) e, igualmente, nas suas competências funcionais. Contribuir para a "inclusão social" significa promover os conceitos de igualdade, direitos humanos e coesão social, de forma que sejam ultrapassados os obstáculos que impedem os indivíduos de participarem ativamente de forma significativa na sociedade (Ronzi et al., 2018).

Por outro lado, é facto que quem apresenta melhores condições económicas parece revelar níveis mais baixos de solidão, quer no período pré quer no pós COVID (Baarck et al., 2021). Tanto a solidão como o isolamento social são, portanto, cada vez mais reconhecidos como questões críticas de saúde pública que merecem atenção e precisam ser abordadas com estratégias de intervenção eficazes (Whitehead \& Torossian, 2021).

A importância do suporte familiar e comunitário é fundamental para os idosos poderem permanecer nos seus domicílios, durante mais tempo e com qualidade (Cabral, 2013). Quando este suporte não é suficiente, os centros de dia ou outras atividades correspondem a uma resposta satisfatória, embora nem sempre, para providenciar atividades diferenciadas e recreativas, nomeadamente atividades de convívios, de reeducação, informativas, aconselhamento, triagem de problemas de saúde, entre outras (Fletcher, 2011; Pender et al., 2015).

Sendo o Envelhecimento tema contemporâneo de debate, as organizações mundiais e outras entidades têm como prioridade a criação de condições mais adequadas para promover melhor qualidade de vida aos mais velhos, mantendo a sua participação no contexto de vida, com segurança (OMS, 2015; Canhestro \& Basto, 2016). Nesta linha de ação mundial, Portugal adotou a Estratégia Nacional para o Envelhecimento Ativo e Saudável (ENEAS) 2017-2025, com o intuito de desenvolver propostas transversais face ao envelhecimento (DGS, 2017) que possam ser aplicadas a nível nacional e que visem a promoção do envelhecimento com direitos, dando poder de autodeterminação às 
pessoas e que, fundamentalmente, se prestem cuidados a essas pessoas na articulação entre as diferentes estruturas de cuidados de saúde e de âmbito social - Resolução da Assembleia da República n88/ 2018.

0 futuro das gerações só é possível quando é considerada a experiência e conhecimento das necessidades dos idosos, designadamente a solidão e isolamento (DGS, 2017) sendo essencial para promover a contribuição da sua participação e manter o seu bem-estar, qualidade de vida, autonomia e independência (UNECE, 2012; DGS, 2017). É com estas premissas que a SCMMC pretende implementar a sua ação na comunidade através de projetos sociais, com intervenção da Música.

\subsection{A Intervenção no contexto rural através da Música}

Segundo alguns estudos, como Bath and Deeg (2005), foi demonstrado que as pessoas que frequentavam atividades de lazer e sociais, evidenciavam melhor saúde mental e física, quando comparadas com idosos que passavam maior parte do seu tempo em casa sozinhos (Sutcliffe et al., 2020), verificando-se uma correlação positiva entre 0 envolvimento social e a perceção de saúde nos domínios psicológico, emocional, social, físico, espiritual e intelectual (Bath \& Deeg, 2005; Zainab \& Naz, 2017). Assim, há evidência que défices associados ao processo de envelhecimento, podem ser prevenidos ou atrasados com intervenções que incluam o uso e treino de várias funções cognitivas, motoras e sensoriais, adaptadas a cada indivíduo. Por meio de atividades físicas e cognitivas estimulantes, prevê-se uma intervenção adequada e positiva com as pessoas mais velhas (Khalsa \& Perry, 2017).

Nesta linha de pensamento, a participação ativa e a capacidade funcional surgem assim como novas componentes no modelo de saúde, uma vez que instalada a incapacidade esta pode determinar a dependência parcial ou total da pessoa em questão e a necessidade de alguém para a auxiliar no seu desempenho, seja ele prestador de cuidados formal ou informal. A promoção e manutenção da saúde mental e física do indivíduo e a prevenção, avaliação e tratamento de todas as formas de perturbação mental e física, podem ser usadas e/ ou podem contribuir para aliviar os défices que se vão instalando (Ribeiro, 2011).

As atividades que visem a exploração da criatividade promovem a inclusão social através da manutenção ou do aumento dos laços entre a pessoa e a comunidade envolvente, melhorando o seu bem-estar psicossocial (Greaves \& Farbus, 2006; Moody \& Phinney, 2012). Por exemplo, o canto é uma forma de atividade musical de intervenção comunitária que melhora significativamente a saúde mental e física dos mais velhos (Skingley \& Bungay, 2010).

Alguns estudos sugerem que a intervenção baseada em atividades musicais pode ter um impacto positivo no bem-estar, na perceção de saúde, qualidade de vida e na saúde física 
e mental (Hallan et al., 2016). Outros estudos indicam que estas intervenções fortalecem os relacionamentos sociais, melhoram a auto-confiança e a auto-estima, assim como os sentimentos de valorização pessoal e a perceção de um estado de saúde física mais ativo, e reduzem o sentimento de isolamento (Ronzi et al., 2018; Sutcliffe et al., 2020). Segundo Veblen (2008), o bem-estar individual e social é, na implementação de atividades musicais na comunidade, tão ou mais importante que a própria aprendizagem musical dos participantes. Também a Alzheimer Portugal (2021) refere no seu site online que a música e as canções revelam ser reminiscências com significado fundamental, uma vez que ativa a memória a longo prazo. A música pode promover o relaxamento, boas lembranças e bem-estar, tendo ainda um efeito positivo em pessoas com sintomatologia depressiva. Pese embora seja necessária mais evidência científica, numa revisão sistemática concluiuse que, de 5 estudos, 4 evidenciavam que a intervenção de reminiscência pela música produzia efeitos positivos nos participantes (Istvandity, 2017).

Atualmente sabe-se que a música recruta praticamente todas as funções cognitivas (Zatorre, 2005) e que esta tem um papel importante na redução efetiva do atraso da deterioração cognitiva nos idosos (Chu et al., 2014). A música promove o envolvimento da componente motora, multissensorial, memória, atenção e emoção (Kraus et al., 2014) sendo, por isso, considerada uma terapêutica holística (Solanki et al. 2013; Carvalho, 2018).

Por fim, a literatura tem vindo corroborar que a arte, utilizada como meio de intervenção, visa facilitar a comunicação e as relações entre as pessoas envolvidas, pois estas agem juntas no mesmo contexto, com objetivos iguais (Zhao et al., 2016). Não obstante, acrescenta-se que a implementação deste tipo de projetos com recurso à arte, nomeadamente à música, pressupõe uma rede de cooperação entre as diferentes entidades sociais e os seus profissionais. Só assim é possível alcançar soluções inovadoras e sustentáveis (Marques, 2013).

\section{METODOLOGIA}

A crescente preocupação de uma Entidade de Responsabilidade Social, como a Santa Casa da Misericórdia de Marco de Canaveses (SCMMC), em prestar mais e melhores cuidados de saúde e apoio social à comunidade, num período de reestruturação decorrente da COVID19, promoveu a necessidade de uma intervenção mais intensa na comunidade Marcoense. Neste âmbito, foram elaborados dois Projetos na área da Saúde Social, baseados na arte musical. Ao longo destes anos tem vindo a ser realizada uma intervenção integrada na linha de intervenção do "ageing in place" sendo constatada a efetiva satisfação e pertinência desta junto da população mais frágil e com pouca 
retaguarda social/económica. A abordagem que abraça estes dois novos Projetos recentemente em implementação apresenta uma perspetiva biopsicossocial centrada nas pessoas idosas, na medida em que o contexto, e tudo o que o envolve, influencia e tem um impacto preponderante na forma como as mesmas vivem, se relacionam, participam e desempenham os seus papéis nas atividades e ocupações do seu dia-a-dia (Ferreira, 2011).

Os projetos foram elaborados e submetidos a financiamento competitivo em diferentes candidaturas:

a) "Bairros Saudáveis", no âmbito do Plano de Recuperação e Resiliência do Governo Português; título do Projeto: "Bairros Saudáveis: Incluir para Melhorar".

b) "Apoio a Projetos Sénior - Combate à solidão da população sénior com recurso às Artes" da Fundação Belmiro de Azevedo; título do Projeto "Musicalidade".

Tendo sido atribuído financiamento, após seleção das propostas, a SCMMC tem as condições para promover a sua execução com cofinanciamento das entidades promotoras e em parceria com outras entidades concelhias.

No sentido de avaliar os indicadores e resultados propostos, após pesquisa bibliográfica, a equipa definiu um protocolo de avaliação quantitativa aos beneficiários dos dois projetos, usando os seguintes instrumentos de avaliação: Whoqol Bref; Escala de Ansiedade, Depressão e Stresse; Escala de Bem-Estar Psicológico de Ryff. Os instrumentos identificados encontram-se devidamente validados para a população portuguesa, tratando-se de escalas de avaliação psicológica adaptadas para a população idosa.

Tendo em conta as caraterísticas dos beneficiários dos projetos - pessoas idosas, na sua maioria com pouca escolarização, inseridos em contexto rural, naturalmente resistentes a processos de avaliação extensos de natureza psicológica - optar-se-á pelo seguinte procedimento: constituição de grupos de avaliação independentes; de forma a homogeneizar a amostra; cada grupo será tanto quanto possível equilibrado em termos de variáveis como sexo, idade, escolaridade, estado cognitivo. A cada grupo será administrado um único instrumento de avaliação. Posteriormente, os resultados serão comparados dentro do próprio projeto, mas também serão comparados os resultados dos dois projetos, uma vez que, apesar de incluir populações idosas em ambos os projetos, os beneficiários encontram-se em contextos diários diferentes (frequência em resposta social vs a residir no domicílio).

A avaliação dos resultados será concretizada em três momentos de avaliação: antes do seu início, a meio da implementação e no final da execução de cada projeto. É importante salientar que serão tidas em consideração alterações na constituição da amostra de um momento de avaliação para o seguinte, podendo ser excluídos participantes se o respetivo estado cognitivo se deteriorar. 


\section{OBJETIVOS E DESTINATÁRIOS}

Com base no conhecimento científico atual, nos pressupostos impostos na candidatura do Governo Português (PNN) e no que está delineado na Estratégia Nacional para o Envelhecimento Ativo e Saudável, a SCMMC tem a preocupação de desenvolver projetos que abranjam problemas reais do contexto de atuação e com os quais possa ter um papel de atores de promoção social e da saúde junto dos mesmos.

Estes dois projetos visam focar o processo de Envelhecimento Ativo e Saudável através de intervenções diversas, com recurso à música, que promovam a participação em atividades de estimulação (competências cognitivas, físicas e sensoriais), a literacia em saúde e a melhoria do bem-estar. A intervenção possibilita também a redução do sentimento de solidão e tristeza (Hays et al, 2002; Särkämö, 2018).

Os Projetos Sociais "Bairros Saudáveis: Incluir para Melhorar" e "Musicalidade" destinamse a pessoas com 65 ou mais anos que residam no concelho de Marco de Canaveses. Para além disto, as pessoas devem realizar marcha de forma independente ou com algum produto de apoio, ter movimento ativo dos membros superiores e apresentar orientação temporo-espacial e autopsiquicamente.

Relativamente ao Projeto "Bairros Saudáveis: Incluir para Melhorar" este terá lugar na comunidade, no domicílio e em locais cedidos pelas Juntas de Freguesia parceiras, para quem não frequente nenhuma resposta social do concelho. As atividades do Projeto "Musicalidade" destinam-se a pessoas que integram alguma resposta social, como Estrutura Residencial para Idosos (ERPI) e Centros de Dia. Concretamente, este projeto atuará em três Centros de Dia, uma ERPI e uma Unidade Cuidados Continuados.

\section{RESULTADOS ESPERADOS}

Através dos Projetos implementados pretende-se fortalecer o sentido de pertença na comunidade dos idosos a residir no próprio domicílio e dos idosos inscritos em respostas sociais do concelho de Marco de Canaveses, através de uma intervenção de proximidade, focada na prevenção da saúde mental. Estima-se obter uma diminuição do isolamento e do sentimento de solidão; uma melhoria na perceção sobre a qualidade de vida e no bemestar psicológico e uma diminuição da sintomatologia depressiva, ansiosa e dos níveis de stress; um aumento do número de participação/interação dos idosos envolvidos; bem como contribuir para a discriminação dos fatores positivos de intervenção das atividades musicais, como ação distintiva, na população idosa. 


\section{CONCLUSÃO}

A experiência de vários anos de intervenção dedicada às vidas longas, num cenário rural, tem mostrado a sua contribuição para a melhoria da qualidade de vida e bem-estar, através da implementação de ações multidisciplinares e inclusivas, desenvolvidos por uma instituição da Economia Social - a Santa Casa da Misericórdia de Marco de Canaveses. A estratégia proposta neste trabalho, congrega experiências anteriores de atuação nesta população, priorizando a introdução das Artes como elemento de estímulo ao desenvolvimento de comportamentos de adaptação e promoção cognitiva e sensorial, através da Música.

Com este trabalho pretende-se não só analisar os benefícios de atividades musicais, como constituir uma forma simples de comunicação alternativa e envolver uma expressão individual alicerçada em pressupostos que estimulem a criatividade, funcionando como estratégias de coping. Será permitido aos participantes lidar de forma eficaz com sintomatologia relacionada com ansiedade, depressão e stress, assim como, possibilitar a re-conexão com processos mnésicos, promovendo melhorias a nível geral na qualidade de vida e na sensação de bem-estar psicológico.

A concretização deste trabalho, pretende mostrar-se essencialmente benéfico para a população idosa mais fragilizada, permitindo também atenuar ou melhorar a sua própria aceitação, bem como o desenvolvimento intelectual e artístico, destacando-se a sua função promocional ao envelhecimento ativo e saudável.

Cada vez mais são demonstrados os efeitos positivos da música enquanto terapia - uma simples melodia pode reavivar lembranças de acontecimentos marcantes da vida de uma pessoa. 


\section{BIBLIOGRAFIA}

Baarck, J., Balahur, A., Cassio, L., d'Hombres, B., Pásztor, Z. \& Tintori, G., (2021). Loneliness in the EU - Insights from surveys and online media data, EUR 30765 EN, Publications Office of the European Union, Luxembourg, https://doi:10.2760/28343

Bherer L. (2015). Cognitive plasticity in older adults: effects of cognitive training and physical exercise. Annals of the New York Academy of Sciences, 1337, 1-6. https://doi.org/10.1111/nyas.12682

Correia, S. (2003). Introdução à Gerontologia. Lisboa: Universidade Aberta.

Bath, P. \& Deeg, D. (2005). Social engagement and health outcomes among older people: introduction to a special section. European Journal of Ageing, 2(1), 24-30. https:// doi:10.1007/s10433-005-0019-4

Cabral, M., Ferreira, P., Silva, P., Jerónimo, P., \& Marques, T. (2013). Processos de envelhecimento em Portugal: usos do tempo, redes sociais e condições de vida. Lisboa: Fundação Francisco Manuel dos Santos.

Canhestro, S. \& Basto, L. (2016). Envelhecer com Saúde: Promoção de Estilos de Vida Saudáveis no Baixo Alentejo. Pensar Enfermagem, 20. 27-51.

Carvalho, B. (2018) Efeitos da Musicoterapia na Agitação em Idosos com Demência - uma Revisão Sistemática [Master's thesis, Politécnico do Porto]. Repositório Científico do Instituto Politécnico do Porto

Chu, H., Yang, C.-Y., Lin, Y., Ou, K.-L., Lee, T.-Y., O’Brien, A. P., \& Chou, K.-R. (2014). The Impact of Group Music Therapy on Depression and Cognition in Elderly Persons With Dementia: A Randomized Controlled Study. Biological Research for Nursing ,16(2), 209-217. https://doi.org/10.1177/1099800413485410

Direção-Geral da Saúde (2017). Estratégia Nacional para o Envelhecimento Ativo e Saudável (ENEAS) 2016-2025 (Despacho $n^{0} \quad$ 12427/2016). https://www.sns.gov.pt/wp-content/uploads/2017/07/ENEAS.pdf

Stanhope, M. \& Lancaster, J. (2011). Enfermagem de Saúde Pública - Cuidados de Saúde na Comunidade centrados na população. (7 ${ }^{\mathrm{a}}$ ed.). (697- 716). Loures: Lusodidacta.

Ferreira, P. (2011). Envelhecimento activo e relações intergeracionais. XV Congresso Brasileiro de Sociologia. Instituto de Ciências Socias da Universidade de Lisboa.

Gai, J., Gomes, L. \& Jansen de Cárdenas, C. (2009). Ptofobia - 0 Medo de Cair em Pessoas Idosas. Acta Médica Portuguesa, 22, 83-88. 
Goldspink, G., \& Harridge, S. D. (2004). Growth factors and muscle ageing. Experimental gerontology, 39(10), 1433-1438. https://doi.org/10.1016/j.exger.2004.08.010

Greaves, Colin J., \& Farbus, L. (2006). Effects of creative and social activity on the health and well-being of socially isolated older people: outcomes from a multi-method observational study. The Journal of The Royal Society for the Promotion of Health, 126(3), 134-142. https://doi.org/10.1177/1466424006064303

Hays, T., Bright, R., \& Minichiello, V. (2002). The Contribution of Music to Positive Aging: A Review. Journal of Aging and Identity, 7(3), 165-175. https://doi:10.1023/a:1019712522302

Hallan, S., Cross, I., \& Thaut, M. (2016). Where now? In S. Hallan, I. Cross \& M. Thaut (Eds.), The Oxford handbook of Music Psychology (2 ed., pp. 905-913). Oxford: Oxford University Press.

Instituto Nacional de Estatística. (2021, Nov 29). Objetivos de Desenvolvimento Sustentável, Agenda 2030. Indicadores para Portugal - 2010/2019. https://www.ine.pt/xportal/xmain?xpid=INE\&xpgid=ine_publicacoes\&PUBLICACOES pub_boui $=434925883 \&$ PUBLICACOESmodo=2\&xlang $=p t$

Istvandity L. (2017). Combining music and reminiscence therapy interventions for wellbeing in elderly populations: A systematic review. Complementary therapies in clinical practice, 28, 18-25. https://doi.org/10.1016/j.ctcp.2017.03.003

Kazazi, L., Foroughan, M., Nejati, V., \& Shati, M. (2018). Association between age associated cognitive decline and health related quality of life among Iranian older individuals. Electronic physician, 10(4), 6663-6671. https://doi.org/10.19082/6663

Khalsa, D. S., \& Perry, G. (2017). The Four Pillars of Alzheimer's Prevention. Cerebrum: the Dana forum on brain science, 2017, cer-03-17.

Kraus, N., Zatorre, R. J., \& Strait, D. L. (2014). Editors' introduction to Hearing Research special issue: music: a window into the hearing brain. Hearing research, 308, 1. https://doi.org/10.1016/j.heares.2013.09.013

Lewis, S. (2003). Elder Care in Occupational Therapy (2ed.). USA: Slack Incorpored.

Longman, J., Passey, M., Singer, J., \& Morgan, G. (2013). The role of social isolation in frequent and/or avoidable hospitalisation: rural community-based service providers' perspectives. Australian health review: a publication of the Australian Hospital Association, 37(2), 223-231. https://doi.org/10.1071/AH12152

Losada, A., Márquez-González, M., García-Ortiz, L., Gómez-Marcos, M. A., FernándezFernández, V., \& Rodríguez-Sánchez, E. (2012). Loneliness and mental health in a 
representative sample of community-dwelling Spanish older adults. The Journal of psychology, 146(3), 277-292. https://doi.org/10.1080/00223980.2011.582523

arioni, R. E., Valenzuela, M. J., van den Hout, A., Brayne, C., Matthews, F. E., \& MRC Cognitive Function and Ageing Study (2012). Active cognitive lifestyle is associated with positive cognitive health transitions and compression of morbidity from age sixty-five. PloS one, 7(12), e50940. https://doi.org/10.1371/journal.pone.0050940

Marques, E. (2013). Intervenção Comunitária através da Arte com pessoas em situação de sem-abrigo. Revista Espacios Transnacionales. doi: http://www.espaciostransnacionales.org

Moody, E., \& Phinney, A. (2012). A community-engaged art program for older people: fostering social inclusion. Canadian journal on aging $=\mathrm{La}$ revue canadienne $\mathrm{du}$ vieillissement, 31(1), 55-64. https://doi.org/10.1017/S0714980811000596

Pender, N. Murdaugh, C. \& Parsons, M. (2015). Health Promotion In: Nursing Practice. ( $7^{\mathrm{a}}$ ed.). Boston: Parsons Education.

Pinto, E. (2011). Independência funcional e regresso ao domicílio: variável chave para a enfermagem de reabilitação. [Master's thesis, Escola Superior de Saúde de Viseu]. https://repositorio.ipv.pt/bitstream/10400.19/1617/1/PINTO\%20Elsa\%20Maria\%20E steves\%20Monteiro\%20Independ\%c3\%aancia\%20Funcional\%20e\%20Regresso $\% 20$ ao\%20Domicilio.pdf

Organização Mundial de Saúde. (2015). Relatório Mundial de Envelhecimento e Saúde. https://apps.who.int/iris/bitstream/handle/10665/186468/WHO_FWC_ALC_15.01_po r.pdf?sequence $=6$

Ribeiro, 0. \& Paúl, C. (2011). Envelhecimento Ativo. In idem (coord.). Manual de Envelhecimento Ativo. Lisboa: Edições Lidel, 1-12.

Ronzi, S., Orton, L., Pope, D., Valtorta, N. K., \& Bruce, N. G. (2018). What is the impact on health and wellbeing of interventions that foster respect and social inclusion in community-residing older adults? A systematic review of quantitative and qualitative studies. Systematic reviews, 7(1), 26. https://doi.org/10.1186/s13643-018-0680-2

Särkämö T. (2018). Cognitive, emotional, and neural benefits of musical leisure activities in aging and neurological rehabilitation: A critical review. Annals of physical and rehabilitation medicine, 61(6), 414-418. https://doi.org/10.1016/j.rehab.2017.03.006

Shearer, N., Fleury, J., Ward, K. A., \& O'Brien, A. M. (2012). Empowerment interventions for older adults. Western journal of nursing research, 34(1), 24-51. https://doi.org/10.1177/0193945910377887 
Skingley, Ann \& Bungay. (2010). The Silver Song Club Project: singing to promote the health of older people. British journal of community nursing, 15(3), 135-140. https://doi.org/10.12968/bjcn.2010.15.3.46902

Sutcliffe, R., Du, K. \& Ruffman, T. (2020). Music Making and Neuropsychological Aging: A Review. Neuroscience Biobehavioral 113, 479-491. https://doi.org/10.1016/j.neubiorev.2020.03.026

United Nations Department of Economic and Social Affairs Population Division. (2015). World population, ageing. New York. doi: ST/ESA/SER.A/390

United Nations Economic Comission for Europe. (2012). Active ageing. UNECE Policy Brief on Ageing, (13).

Veblen, K. (2008). The many ways of community music. International Journal of Community Music, 1(1), 5-21.

Whitehead, B. \& Torossian, E. (2021). Older Adults' Experience of the COVID-19 Pandemic: A Mixed-Methods Analysis of Stresses and Joys. Gerontologist, 61(1), 36-47. https://doi.org/10.1093/geront/gnaa126

World Health Organization. (2015). Measuring the age-friendliness of cities: A guide to using core indicators.

https://apps.who.int/iris/bitstream/handle/10665/203830/9789241509695_eng.pdf?seque nce $=1$ \&isAllowed=y

World Health Organization. (2021). Ageing and health. http://www.who.int/newsroom/fact-sheets/detail/ageing-and-health

Wu, B. (2020). Social isolation and loneliness among older adults in the context of COVID19: a global challenge. Global Health Research and Policy, 5(27). https://doi.org/10.1186/s41256-020-00154-3

Zainab, N. \& Naz, H. (2017). Daily Living Functioning, Social Engagement and Wellness of Older Adults. Psychology, Community \& Health. 6(1), 93-102. https://doi:10.5964/pch.v6i1.213

Zatorre, R. (2005). Music, the food of neuroscience?. Nature, 434, $312-315$.

Zhao, K., Bai, Z. G., Bo, A., \& Chi, I. (2016). A systematic review and meta-analysis of music therapy for the older adults with depression. International journal of geriatric psychiatry, 31(11), 1188-1198. https://doi.org/10.1002/gps.4494 